\title{
The Association Between Home Stay and Symptom Severity in Major Depressive Disorder: Preliminary Findings From a Multicenter Observational Study Using Geolocation Data From Smartphones
}

Petroula Laiou ${ }^{1 *}, \mathrm{PhD}$; Dzmitry A Kaliukhovich ${ }^{2 *}, \mathrm{PhD}$; Amos A Folarin ${ }^{1,3,4,5,6}, \mathrm{PhD}$; Yatharth Ranjan ${ }^{1}$, MSc; Zulqarnain Rashid $^{1}$, PhD; Pauline Conde ${ }^{1}$, BSc; Callum Stewart ${ }^{1}$, MSc; Shaoxiong Sun ${ }^{1}$, PhD; Yuezhou Zhang ${ }^{1}$, MSc; Faith Matcham $^{7}, \mathrm{PhD}$; Alina Ivan ${ }^{7}, \mathrm{MSc}$; Grace Lavelle ${ }^{7}, \mathrm{PhD}$; Sara Siddi ${ }^{8,9,10}$, PhD; Femke Lamers ${ }^{11}$, PhD; Brenda WJH Penninx ${ }^{11}$, PhD; Josep Maria Haro ${ }^{8,9,10}$, PhD; Peter Annas ${ }^{12}$, PhD; Nicholas Cummins ${ }^{1}, \mathrm{PhD}$; Srinivasan Vairavan ${ }^{13}$, $\mathrm{PhD}$; Nikolay V Manyakov ${ }^{2}$, PhD; Vaibhav A Narayan ${ }^{13}, \mathrm{PhD}$; Richard JB Dobson ${ }^{13,4,5,6}, \mathrm{PhD}$; Matthew Hotopf ${ }^{4,7}$, $\mathrm{PhD}$; RADAR-CNS ${ }^{14}$

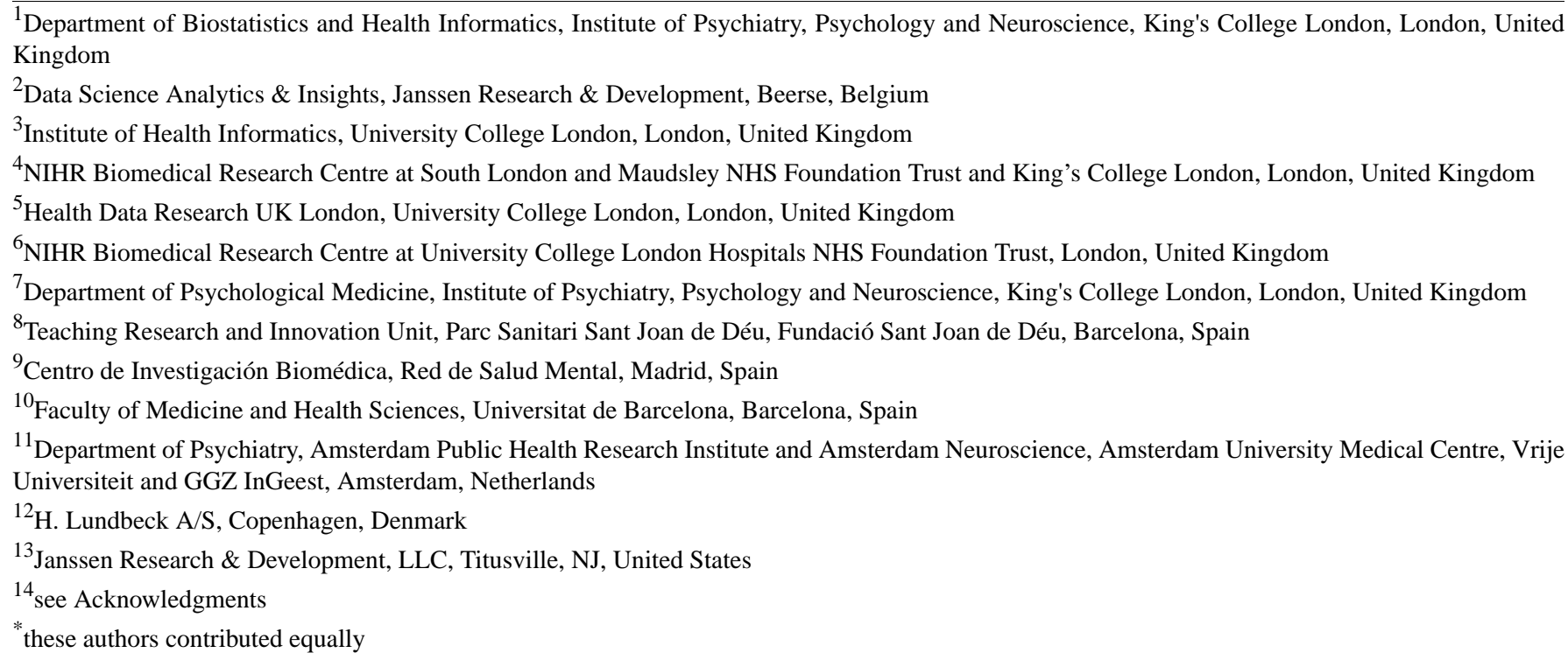

\section{Corresponding Author:}

Petroula Laiou, $\mathrm{PhD}$

Department of Biostatistics and Health Informatics

Institute of Psychiatry, Psychology and Neuroscience

King's College London

Memory Lane

London, SE5 8AF

United Kingdom

Phone: 442078480002

Email: petroula.laiou@kcl.ac.uk

\section{Abstract}

Background: Most smartphones and wearables are currently equipped with location sensing (using GPS and mobile network information), which enables continuous location tracking of their users. Several studies have reported that various mobility metrics, as well as home stay, that is, the amount of time an individual spends at home in a day, are associated with symptom severity in people with major depressive disorder (MDD). Owing to the use of small and homogeneous cohorts of participants, it is uncertain whether the findings reported in those studies generalize to a broader population of individuals with MDD symptoms. 
Objective: The objective of this study is to examine the relationship between the overall severity of depressive symptoms, as assessed by the 8-item Patient Health Questionnaire, and median daily home stay over the 2 weeks preceding the completion of a questionnaire in individuals with MDD.

Methods: We used questionnaire and geolocation data of 164 participants with MDD collected in the observational Remote Assessment of Disease and Relapse-Major Depressive Disorder study. The participants were recruited from three study sites: King's College London in the United Kingdom (109/164, 66.5\%); Vrije Universiteit Medisch Centrum in Amsterdam, the Netherlands (17/164, 10.4\%); and Centro de Investigación Biomédica en Red in Barcelona, Spain (38/164, 23.2\%). We used a linear regression model and a resampling technique $(n=100$ draws) to investigate the relationship between home stay and the overall severity of MDD symptoms. Participant age at enrollment, gender, occupational status, and geolocation data quality metrics were included in the model as additional explanatory variables. The $95 \% 2$-sided CIs were used to evaluate the significance of model variables.

Results: Participant age and severity of MDD symptoms were found to be significantly related to home stay, with older (95\% CI 0.161-0.325) and more severely affected individuals (95\% CI 0.015-0.184) spending more time at home. The association between home stay and symptoms severity appeared to be stronger on weekdays (95\% CI 0.023-0.178, median 0.098; home stay: 25th-75th percentiles $17.8-22.8$, median 20.9 hours a day) than on weekends (95\% CI -0.079 to 0.149 , median 0.052 ; home stay: 25th-75th percentiles 19.7-23.5, median 22.3 hours a day). Furthermore, we found a significant modulation of home stay by occupational status, with employment reducing home stay (employed participants: 25th-75th percentiles 16.1-22.1, median 19.7 hours a day; unemployed participants: 25th-75th percentiles 20.4-23.5, median 22.6 hours a day).

Conclusions: Our findings suggest that home stay is associated with symptom severity in MDD and demonstrate the importance of accounting for confounding factors in future studies. In addition, they illustrate that passive sensing of individuals with depression is feasible and could provide clinically relevant information to monitor the course of illness in patients with MDD.

(JMIR Mhealth Uhealth 2022;10(1):e28095) doi: 10.2196/28095

\section{KEYWORDS}

major depressive disorder; PHQ-8; smartphone; GPS; home stay; mobile phone

\section{Introduction}

The World Health Organization ranks depression as the single largest contributor to global disability [1]. People with major depressive disorder (MDD) often experience physical comorbidity [2], loss of occupational function [3], and low quality of life [4]. Furthermore, MDD is strongly associated with suicidal deaths and premature mortality [5]. The process for MDD diagnosis and evaluation of symptom severity is highly dependent on the subjective information that an individual under screening provides to a clinician, and it might be affected by recall bias.

Recent advances in digital technologies, including smartphones and wearable devices, enable the collection of a variety of data streams that can be used to objectively characterize an individual's daily activity and physical condition [6]. These data can be collected continuously, remotely, and unobtrusively without affecting an individual's daily routine and behavior. Importantly, analysis of such data could result in the development of new objective, quantifiable, cost-effective, and viable digital biomarkers of an individual's behavioral, cognitive, and emotional states [7-9]. Once developed and thoroughly tested, digital biomarkers hold great promise for improving the diagnosis and prognosis of a variety of mental health disorders, for facilitating continuous monitoring of individual well-being, and for supporting initiatives in precision medicine by helping to establish digital patient phenotypes in different disease areas [10].

Several recent studies have demonstrated the association between MDD symptoms and mobility patterns derived from mobile devices. For example, individuals with greater severity of MDD symptoms were reported to make fewer transitions between locations of interest (ie, those frequently visited in the past) and spend more time at home [11-15]. Home stay, an indicator of social disengagement [12], has also been reported to be significantly related to the severity of MDD symptoms [12-14].

Most studies used small and homogeneous cohorts of participants (eg, university students) and were conducted over a short period (eg, several weeks). In this study, we examined the association between the overall severity of MDD symptoms and a measure of daily mobility patterns using data from a larger and more diverse group of participants collected in the Remote Assessment of Disease and Relapse-Major Depressive Disorder (RADAR-MDD) study [16]. The RADAR-MDD study is an observational, longitudinal, prospective study that is currently being conducted at multiple clinical sites spread across several European countries and is part of a wider research program (Remote Assessment of Disease and Relapse-Central Nervous System [17]) to explore the potential of wearable devices to help prevent and treat depression, multiple sclerosis, and epilepsy. We used the 8-item Patient Health Questionnaire (PHQ-8; [18]) total score to measure the severity of MDD symptoms and home stay to describe an individual's daily mobility pattern. Home stay was selected as an interpretable measure of mobility with previous evidence suggesting that it is related to the severity of MDD symptoms [12-14]. In addition, we examined whether the strength of the relationship changes from weekdays to weekends (ie, modulated by changes in daily routine) and can be affected by an individual's demographics and quality of the acquired GPS recordings. We hypothesized 
that higher levels of MDD, as quantified by the PHQ-8, would correspond to a prolonged home stay. In addition, we anticipated that the relationship between the severity of MDD symptoms and home stay would be modulated by changes in daily routine from weekdays to weekends [14]. If the hypotheses were proved to be true, this would provide additional evidence on the use of geolocation data in digital phenotyping [10].

\section{Methods}

\section{Study Population}

Participants were recruited for the RADAR-MDD study from November 2017 to November 2019. The recruited participants were aged $\geq 18$ years and had experienced at least two episodes of MDD in their lifetime, with the most recent episode occurring within the last 2 years. The exclusion criteria included lifetime history of bipolar disorder; schizophrenia; MDD with psychotic features, schizoaffective disorders; history of moderate to severe drug or alcohol dependence within 6 months before enrollment; history of a major medical disease that could affect the participant's ability to be involved in normal daily activities for $>2$ weeks; dementia; and pregnancy. No limitations were applied regarding any treatment that the participants were receiving over the course of the study. Written consent was obtained before the enrollment session, followed by collection of sociodemographic, social environment, and medical history and technology use questionnaires and the Lifetime Depression Assessment Self-Report [19]. Participants with MDD were recruited from three clinical sites: King's College London (KCL) in the United Kingdom; Vrije Universiteit Medisch Centrum (VUMC) in Amsterdam, the Netherlands; and Centro de Investigación Biomédica en Red (CIBER) in Barcelona, Spain (Multimedia Appendix 1, Table S1 and Table 1). More details on the study protocol can be found in the study by Matcham et al [16].

Table 1. Data set characteristics ( $\mathrm{N}=164)$.

\begin{tabular}{|c|c|c|c|c|}
\hline \multirow[t]{2}{*}{ Characteristic } & \multicolumn{4}{|l|}{ Clinical site } \\
\hline & $\mathrm{KCL}^{\mathrm{a}}$ & CIBER $^{b}$ & VUMC $^{\mathrm{c}}$ & All sites \\
\hline Participants with both PHQ- $8^{\mathrm{d}}$ and GPS data collected, n (\%) & $232(57.9)$ & $116(28.9)$ & $53(13.2)$ & $401(100)$ \\
\hline $\begin{array}{l}\text { Participants with biweekly segments fulfilling the selection criteria, } \\
\text { n (\%) }\end{array}$ & $109(66.4)$ & $38(23.2)$ & $17(10.4)$ & $164(100)$ \\
\hline Female, $\mathrm{n}(\%)$ & $83(76.1)$ & $26(68.4)$ & $14(82.4)$ & $123(75)$ \\
\hline Age (years), median (range; SD) & $46(18-73 ; 15.0)$ & $54(27-71 ; 9.8)$ & $33(19-69 ; 14.9)$ & $\begin{array}{l}48(18-73 ; \\
14.7)\end{array}$ \\
\hline Biweekly segments analyzed, n (\%) & $483(62.8)$ & $222(28.9)$ & $64(8.3)$ & $769(100)$ \\
\hline For employed participants & $277(72.5)$ & $64(16.8)$ & $41(10.7)$ & $382(100)$ \\
\hline For unemployed participants & $204(53)$ & $158(41)$ & $23(6)$ & $385(100)$ \\
\hline
\end{tabular}

${ }^{\mathrm{a}} \mathrm{KCL}$ : King's College London.

${ }^{\mathrm{b}}$ CIBER: Centro de Investigación Biomédica en Red.

${ }^{c}$ VUMC: Vrije Universiteit Medisch Centrum.

${ }^{\mathrm{d}}$ PHQ-8: 8-item Patient Health Questionnaire.

The number of participants and biweekly segments collected at each site was normalized by the corresponding total obtained by pooling data across the 3 sites (column All sites in Table 1), with the resulting percentages indicated in parentheses. In all, 2 biweekly segments from KCL had no data on occupational status.

\section{Data Collection}

We used the RADAR-based platform for data collection and storage [20,21]. Participants with MDD were required to install several apps on their smartphones. Participants without a smartphone or with a non-Android device were provided with an Android smartphone and were required to use it throughout the study [16]. Remote monitoring technology (RMT) apps were used to collect data on participant severity of experienced MDD symptoms, self-esteem, cognitive functioning, voice audio sampling, and brief in-the-moment assessments of daily life experiences. Specifically, every 2 weeks, the participants were requested to fill in the PHQ- 8 in the RADAR-base active RMT

app. The request notifications were sent out at a calendared time and remained active only on that day initially. The completion window was increased to 3 days to improve the completion rates in April 2019.

The passive RMT apps ran in the background and required minimal input from the participants. The apps collected data on participants' physical (eg, transitions in space) and socially relevant activity (eg, number and duration of phone calls) as well as on some ambient factors (eg, ambient noise and light). GPS location data were obfuscated by adding a fixed random number to the latitude and longitude of all GPS data points generated by a single participant (Figure 1). The accuracy of each acquired GPS data point, as provided by either a mobile network operator or GPS satellites, corresponded to 1 SD (ie, radius) of a bivariate normal distribution with equal variances along the 2 spatial dimensions centered at that point. GPS data points with an accuracy $>20$ meters were discarded from the analyses. This accuracy level allowed the inclusion and analysis of most of the generated biweekly segments while ensuring 
high accuracy of the GPS recordings (Multimedia Appendix 1, Table S2). The sampling period of the GPS signal was set to either 5 minutes [13,14,22] or 10 minutes [23-25] throughout the study. However, the effective sampling period varied over time towing to occasional signal loss and battery drain (ie, signal undersampling). Other factors affecting the sampling period included occasional concurrent and asynchronous acquisition of geolocation data from both a mobile network operator and GPS satellites (ie, signal oversampling).

Figure 1. Exemplar geolocation data which correspond to a biweekly segment of a study participant. The red dots denote individual's home location, whereas longitude and latitude along the axes are expressed in decimal degrees.

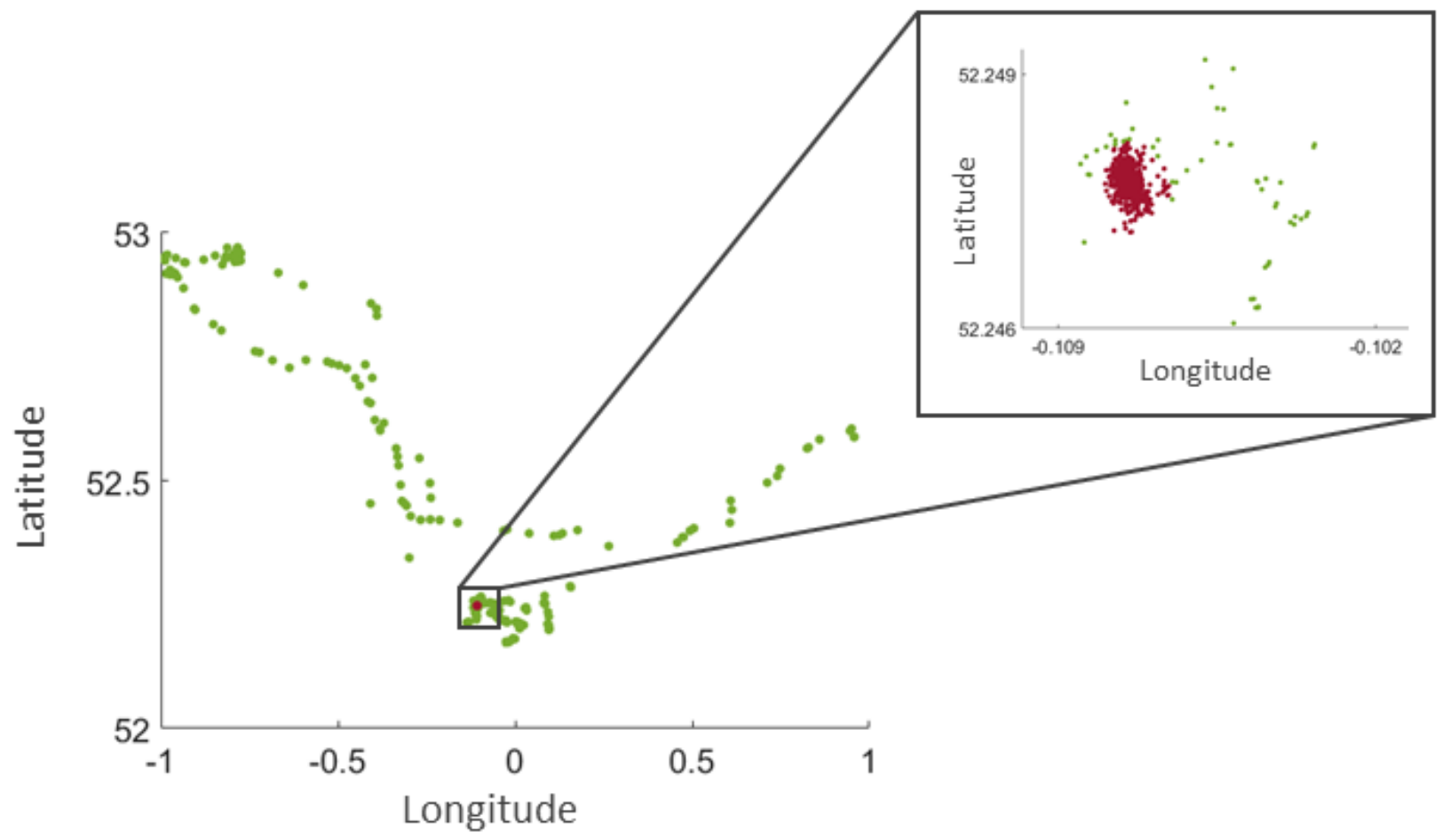

A single completed PHQ-8 combined with the GPS data acquired over the 2 preceding weeks and obtained from the same participant is herein referred to as a biweekly segment. To ensure a high quality of the analyzed geolocation data, only biweekly segments that met the following criteria were analyzed: 14 days of GPS recordings available, a daily median sampling period of the GPS signal $\leq 11$ minutes, and the daily number of acquired GPS data points $\geq 48$. The cutoff values were selected to maximize the volume of data available for analysis while preserving the high quality of these data (Multimedia Appendix 1, Tables S2-S4). Participants who declared their occupational status as a volunteer, student, caregiver, full- or part-time employee, or self-employed person were considered employed, whereas all other participants were considered unemployed. To avoid interference from the effect of the COVID-19 pandemic, only data collected before January 1, 2020, were analyzed.

For each day in a biweekly segment, we computed the number of GPS data points collected for each of the 24 hours separately and over the entire day. Ideally, a GPS signal sampled uniformly over a period of 5 minutes would give 12 GPS data points per hour and a total of 288 GPS data points per day. We specified completeness of the daily data as a ratio between the actual number of GPS data points collected over a day and the expected number as determined by a sampling period (ie, 288 and 144 GPS data points for the sampling periods of 5 minutes and 10 minutes, respectively). The extreme completeness values of 0.0 and 1.0 correspond to an empty and a complete day of GPS recordings, respectively, with the values in-between corresponding to partial or interrupted GPS recordings throughout a day (Multimedia Appendix 1, Table S5). Multimedia Appendix 1, Figure S1 shows the median data completeness for each hour in a day across the analyzed biweekly segments. Similarly, we divided the actual number of GPS data points collected per hour by the expected number and computed the SD of these 24 normalized values to characterize the sampling constancy of the daily data. Any positive value for sampling constancy indicates fluctuations in the volume of GPS data acquired throughout the day, with greater values indicating greater fluctuations (Multimedia Appendix 1, Table S6). Median completeness and median sampling constancy of the daily data, as computed across 14 days of a biweekly segment, were used to characterize the quality of GPS recordings acquired for that segment.

\section{Home Stay}

Home location was identified in a stepwise manner. Initially, the home location was approximated by the median longitude and latitude of all GPS data points in a biweekly segment acquired between 12 AM and 6 AM [12-14]. To account for accidental travel and outdoor stay, all GPS data points acquired during these hours and separated by $>60$ meters $(=3 \times$ the accuracy level of $\leq 20$ meters; see the Data Collection section) from the initial home location were discarded. The home location was finally determined as the median longitude and latitude of all remaining GPS data points (Figure 1). The 
distance between any 2 GPS data points $i$ and $j$ was computed using the Haversine WGS84 formula as follows [22]:

$$
\text { distance }=\sin ^{2}(\Delta \varphi / 2)+\cos \varphi \mathrm{i} \cos \varphi \mathrm{j} \sin ^{2}(\Delta \lambda / 2)
$$

where $\varphi$ and $\lambda$ correspond to the latitude and longitude, respectively.

The home stay for a given day was specified as the ratio between the number of GPS data points separated by $\leq 60$ meters from the home location and the total number of GPS data points acquired on that day. Home stay values of 0 (or $0 \%$ ) and 1 (or $100 \%$ ) correspond to an entire day spent outside versus at home, respectively. Median home stay, as computed across 14 days of a biweekly segment, was used to characterize the home stay of a study participant for that segment (Multimedia Appendix 1, Table S7).

\section{Statistical Analysis}

A linear regression model was selected to test the relationship between home stay and overall severity of MDD symptoms. Specifically, home stay was used as a dependent variable, with PHQ-8 total score being used as an independent variable. Participant age at enrollment, gender (men vs women), occupational status (employed vs unemployed), median completeness, and sampling constancy of the daily data in a biweekly segment were included in the model as additional explanatory variables:

\section{home stay $\approx \mathrm{PHQ}-8$ score+age+gender+occupational} status+data completeness+sampling constancy

We chose home stay as a dependent variable to test its relationship not only to the severity of MDD symptoms but also to participants' demographics and quality characteristics of the collected geolocation data in a single model in a uniform manner. For each study participant, we randomly selected one of the biweekly segments generated by that participant. The model was fitted using data from biweekly segments pooled across the participants. To obtain a CI for each of the 6 regression coefficients, the procedure of random selection of a biweekly segment per participant followed by pooling data across the participants and fitting the model was repeated 100 times. A model variable was deemed to be significantly related to home stay if a 95\% 2-sided CI obtained for the regression coefficient of that parameter did not include 0 . The model was fitted using data from all 3 sites combined (Table 2) and each clinical site separately (Multimedia Appendix 1, Table S8).

Table 2. CIs and medians for the 6 regression coefficients of the linear regression model.

\begin{tabular}{|c|c|c|c|c|c|c|}
\hline \multirow{2}{*}{$\begin{array}{l}\text { Analyzed time } \\
\text { frame }\end{array}$} & \multicolumn{6}{|c|}{ Value, median $(95 \% \mathrm{CI})$} \\
\hline & Age & Gender & $\begin{array}{l}\text { PHQ-8 }{ }^{\mathrm{a}} \text { total } \\
\text { score }\end{array}$ & Occupational status & $\begin{array}{l}\text { Median completeness of } \\
\text { the daily data }\end{array}$ & $\begin{array}{l}\text { Median sampling constan- } \\
\text { cy of the daily data }\end{array}$ \\
\hline $\begin{array}{l}\text { Over the entire } \\
\text { week }\end{array}$ & $\begin{array}{l}0.241(0.161 \text { to } \\
0.325)^{\mathrm{b}}\end{array}$ & $\begin{array}{l}-0.121(-0.272 \\
\text { to } 0.024)\end{array}$ & $\begin{array}{l}0.100(0.015 \text { to } \\
0.184)\end{array}$ & $\begin{array}{l}-0.448(-0.631 \text { to } \\
-0.279)\end{array}$ & $-0.044(-0.108$ to 0.022$)$ & $-0.064(-0.130$ to 0.005$)$ \\
\hline Weekdays only & $\begin{array}{l}0.254(0.188 \text { to } \\
0.329)\end{array}$ & $\begin{array}{l}-0.061(-0.220 \\
\text { to } 0.058)\end{array}$ & $\begin{array}{l}0.098(0.023 \text { to } \\
0.178)\end{array}$ & $\begin{array}{l}-0.495(-0.664 \text { to } \\
-0.354)\end{array}$ & $-0.024(-0.097$ to 0.034$)$ & $-0.041(-0.110$ to 0.041$)$ \\
\hline Weekends only & $\begin{array}{l}0.148(0.029 \text { to } \\
0.240)\end{array}$ & $\begin{array}{l}-0.036(-0.265 \\
\text { to } 0.174)\end{array}$ & $\begin{array}{l}0.052(-0.079 \\
\text { to } 0.149)\end{array}$ & $\begin{array}{l}-0.323(-0.535 \text { to } \\
-0.127)\end{array}$ & $0.023(-0.074$ to 0.101$)$ & $-0.075(-0.144$ to 0.006$)$ \\
\hline
\end{tabular}

${ }^{\mathrm{a}}$ PHQ-8: 8-item Patient Health Questionnaire.

${ }^{\mathrm{b}} \mathrm{CIs}$ that do not include 0 are italicized. The regression coefficients obtained with standardized data for each clinical site separately are reported in Multimedia Appendix 1, Table S8.

The model was fitted using standardized data pooled across the 3 sites for each analyzed time frame separately. The positive sign of the regression coefficients that correspond to the categorical variables (ie, gender and occupational status) indicates greater home stay for men and employed as compared with women and unemployed participants, respectively. All reported CIs are $95 \%$ 2-sided intervals.

To test whether the relationship between home stay and the independent variables differed between weekdays and weekends, a similar approach was followed. Specifically, home stay, median completeness, and sampling constancy of the daily data in a biweekly segment were estimated separately for weekdays and weekends. As a single biweekly segment included 10 weekdays and only 4 weekend days, we used 4 days to generate those estimates to equalize variance in the estimates of weekdays and weekends. For each analyzed biweekly segment, we randomly drew 4 weekdays 100 times. The medians of the estimates computed for each of these 100 draws were used to characterize the weekdays of that segment in the model.

To account for nonnormality of both dependent and independent variables as well as for differences in their variance, each variable (except for gender and occupational status) was standardized by applying the Yeo-Johnson transformation followed by the zero-mean, unit-variance normalization. All models and findings reported throughout the manuscript were obtained by using these standardized data. However, qualitatively similar results were obtained when using the original, nonstandardized data (Multimedia Appendix 1, Table S9). All statistical analyses were performed using the Matrix Laboratory R2019b. 


\section{Results}

\section{Data Set Characteristics}

As of January 1, 2020, the total number of participants enrolled in the RADAR-MDD study across the 3 clinical sites was 432 (Multimedia Appendix 1, Table S1). Of those 432, a total of $401(92.8 \%)$ participants had usable PHQ-8 and geolocation data (Table 1), resulting in a total of 4273 biweekly segments generated across the sites (Multimedia Appendix 1, Table S2). After discarding GPS data points with low accuracy $(>20$ meters) and selecting only biweekly segments with 14 days of GPS recordings available, the number of biweekly segments reduced to 43.9\% (1876/4273; Multimedia Appendix 1, Table S2). Imposing additional requirements on the daily median sampling period ( $\leq 11$ minutes; Multimedia Appendix 1, Table S3) and the daily minimum volume ( $\geq 48$ data points; Multimedia Appendix 1, Table S4) of the GPS data in a single biweekly segment further reduced the number of biweekly segments available for analysis to $17.99 \%$ (769/4273; Table 1$)$. The latter corresponds to data from $38 \%$ (164/432) of study participants. Table 1 lists the demographic characteristics of the participants, whereas Figure 2C shows the distribution of their ages. Most of the study participants enrolled at each clinical site were women (range 26/38, 68\%-14/17, 82\%; Table 1; [26]).

Figure 2. Distributions of data set characteristics. (A) Number of biweekly segments available for analysis per study participant. (B) 8-item Patient Health Questionnaire total score. (C) Participant age. Data were pooled across the 3 clinical sites. PHQ-8: 8-item Patient Health Questionnaire.
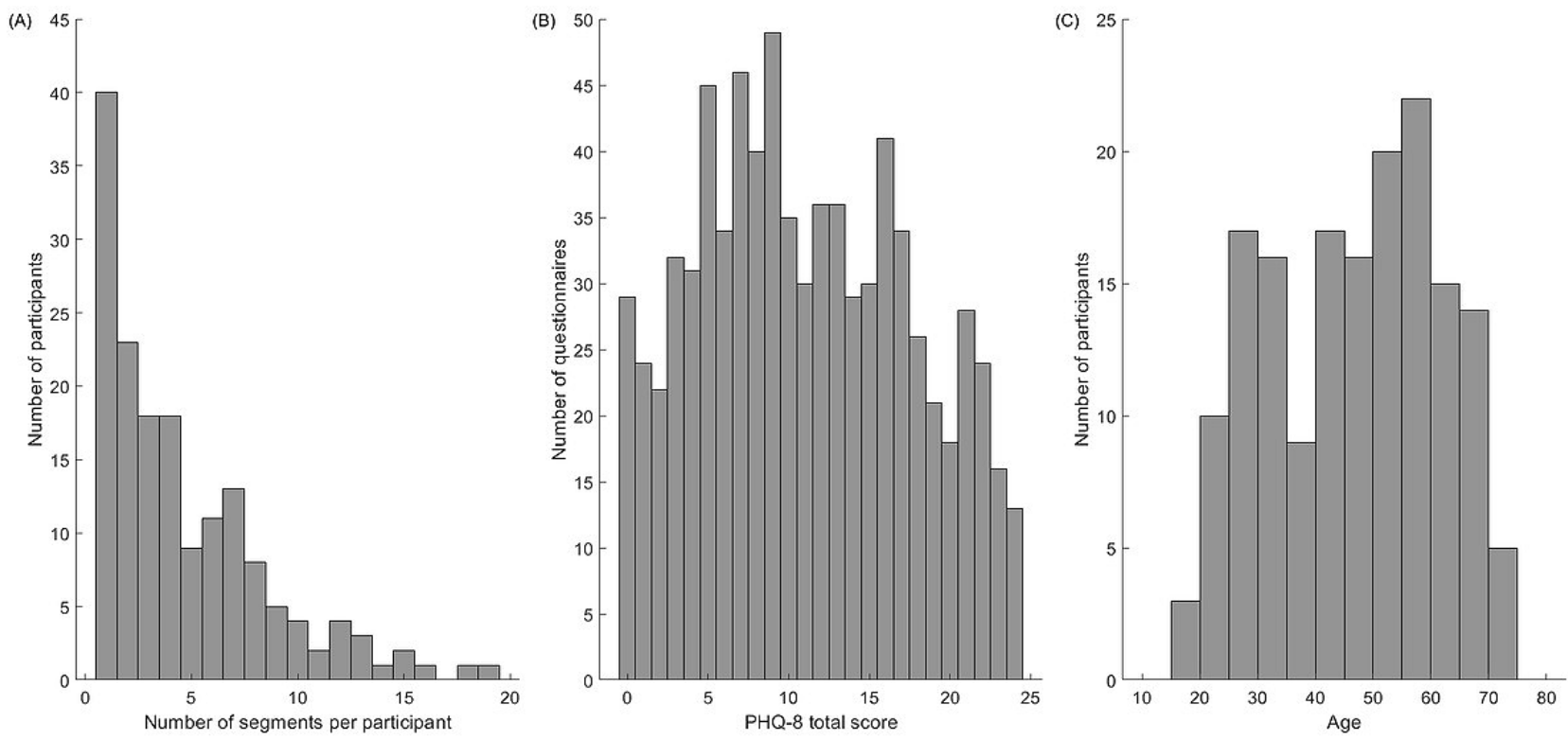

The number of biweekly segments available for analysis varied considerably across the sites, with VUMC (64/769, 8.3\% segments; Table 1) and KCL $(483 / 769,62.8 \%)$ providing the least and most data, respectively. The number of biweekly segments produced by a single participant varied between 1 and 19 , with a median equaling 4 (25th-75th percentiles $2-7$; Figure 2A). As shown in Figure 2B, the collected data represented all 5 severity categories of MDD, as specified in the PHQ-8 questionnaire [18], including none-minimal (PHQ-8 total score from 0 to $4 ; 138 / 769,18 \%$ segments), mild (PHQ-8 total score from 5 to $9 ; 214 / 769,27.8 \%$ of the total), moderate (PHQ-8 total score from 10 to $14 ; 166 / 769,21.6 \%$ of the total), moderately severe (PHQ-8 total score from 15 to $19 ; 152 / 769$, $19.8 \%$ of the total), and severe (PHQ-8 total score from 20 to $24 ; 99 / 769,12.8 \%$ of the total). The data set characteristics for each individual site and occupational status are shown in Multimedia Appendix 1, Figures S2 and S3.

\section{Estimates of Home Stay}

Over the course of the study, the participants spent most of their time at home. When no distinction between weekdays and weekends was made, median home stay across the sites was $89 \%$ (21.4 hours a day; 25th-75th percentiles $76 \%-96 \%$ or 18.2-23.0 hours a day; Figure 3A). As expected, the home stay was lower during weekdays than during the weekends (Figures $3 \mathrm{~B}$ and $3 \mathrm{C})$. Specifically, the median home stay across the sites was $87 \%$ (20.9 hours a day; 25 th- 75 th percentiles $74 \%-95 \%$ or 17.8-22.8 hours a day) and 93\% (22.3 hours a day; 25th-75th percentiles $82 \%-98 \%$ or $19.7-23.5$ hours a day) when analyzing weekday and weekend data, respectively. These observations were consistent across each clinical site (Multimedia Appendix 1, Figure S4 and Table S7). 
Figure 3. Home stay computed (A) over the entire week, (B) for weekdays, and (C) weekends only. A grey horizontal bar and a cross in each boxplot indicate median and mean of the presented data. Data were pooled across the 3 clinical sites. Home stay corresponds to the percentage of time spent at home a day.
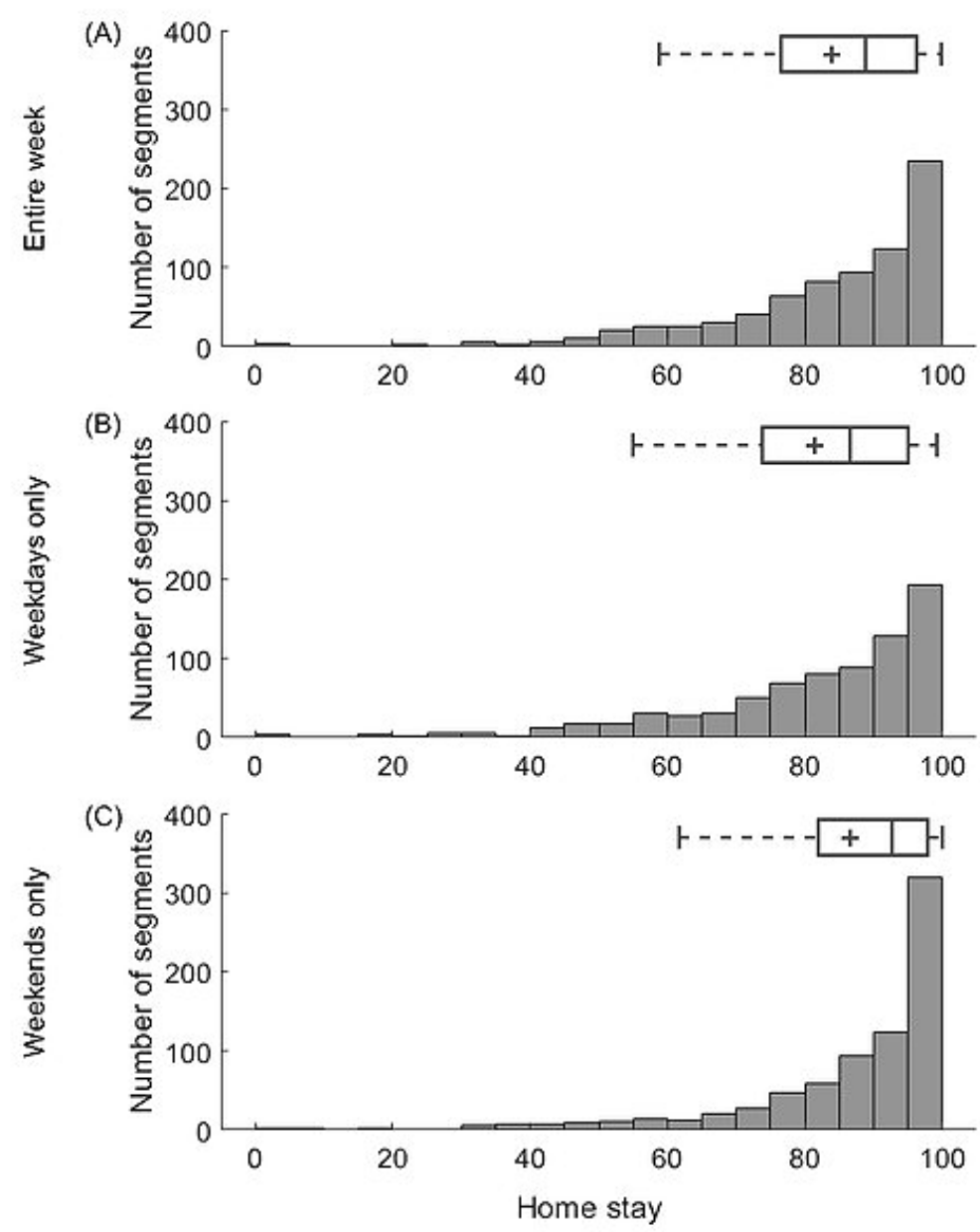

Similarly, home stay was affected by occupational status. The employed participants spent less time at home compared with their unemployed peers. Median home stay across the sites was $82 \%$ (19.7 hours a day; 25th-75th percentiles $67 \%-92 \%$ or 16.1-22.1 hours a day) and $94 \%$ (22.6 hours a day; 25 th-75th percentiles $85 \%-98 \%$ or $20.4-23.5$ hours a day) for the employed and unemployed participants, respectively, with the difference being more prominent during weekdays (79\% vs $93 \%$ or 19.0 vs 22.3 hours a day) than during the weekends ( $88 \%$ vs $96 \%$ or 21.1 vs 23.0 hours a day). The same pattern of observations was seen across each clinical site (Multimedia Appendix 1, Figure S5 and Table S7).

\section{Associations With Home Stay}

When data were pooled across the sites and no distinction between weekdays and weekends was made, the linear regression model revealed a significant relationship between home stay and overall severity of the MDD symptoms as captured by the PHQ-8 total score (median 0.100, 2-sided 95\% CI 0.015-0.184; Figure 4D; Table 2). The latter suggested that greater overall severity of MDD symptoms was associated with prolonged home stay. The same relationship was observed when analyzing weekday data only (median 0.098, 95\% CI
0.023-0.178; Figure 4E) but not on weekends (median 0.052, $95 \%$ CI -0.079 to 0.149 ; Figure $4 \mathrm{~F}$ ).

In addition, the model revealed a significant relationship between home stay and age. Specifically, the participants spent more time at home with age (median 0.241, 95\% CI 0.161-0.325; Figure 4A; Table 2). A similar strength of the relationship was observed for weekdays (median $0.254,95 \%$ CI 0.188-0.329; Figure 4B) and weekends (median 0.148, $95 \%$ CI 0.029-0.240; Figure 4C). Furthermore, occupational status was also found to significantly modulate home stay, with the employed participants spending less time at home compared with their unemployed peers (median -0.448 , 95\% CI -0.631 to -0.279 ; Table 2). Similar to age, there was no significant difference in the effect of occupational status on home stay among the analyzed time frames (weekdays only: median $-0.495,95 \%$ CI -0.664 to -0.354 ; weekends only: median $-0.323,95 \%$ CI -0.535 to -0.127 ).

Neither gender nor median completeness and sampling constancy of the daily data in a biweekly segment had a significant impact on home stay and this held for all the analyzed time frames (Table 2). The results of modeling for each clinical site obtained with standardized and original data are shown in Multimedia Appendix 1, Tables S8 and S9, respectively. 
Figure 4. Relationship (A, B, and C) between home stay and participant age and (D, E, and F) between home stay and the 8-item Patient Health Questionnaire total score as assessed using data of (A and D) the entire week, (B and E) weekdays, and (C and F) weekends only. Each dot indicates a single biweekly segment. Data of all biweekly segments pooled across the 3 clinical sites are presented. A black line in each panel corresponds to the linear fit of the presented data. Home stay corresponds to the percentage of time spent at home a day. PHQ-8: 8-item Patient Health Questionnaire.
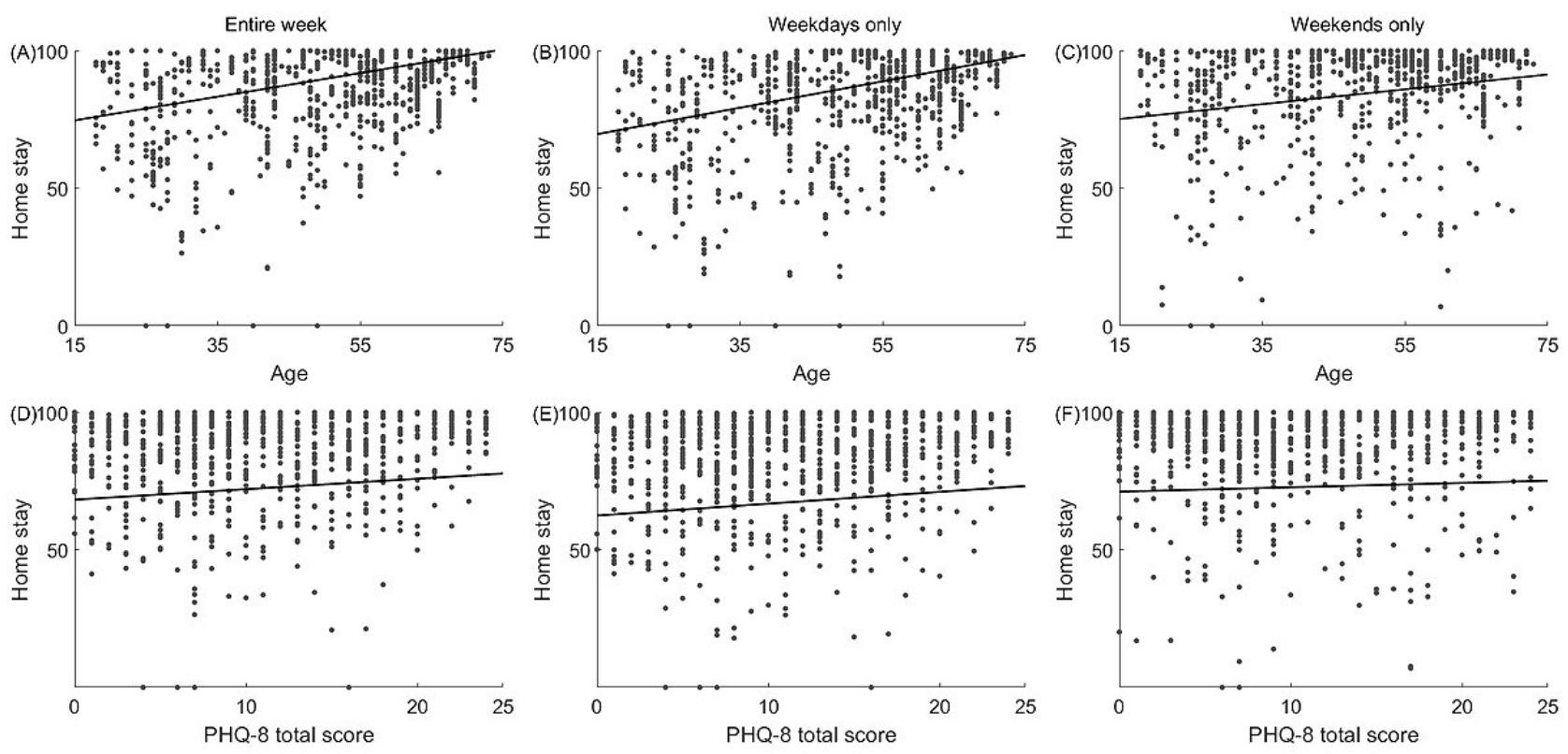

\section{Discussion}

\section{Principal Findings}

Multiple studies have demonstrated associations between patterns of daily movements of an individual in an area of the primary residence and an individual's mood [9]. Here, we tested the association between home stay and overall severity of MDD symptoms, as reflected in the PHQ-8 total score, by using data collected in the RADAR-MDD study. The participants were invited to complete the PHQ-8 on their mobile phones every 2 weeks, whereas the same phones were used to track their geographic location continuously throughout the study. We related the PHQ-8 total score, as provided by an individual, to their median daily home stay over the 2 weeks preceding completion of the PHQ-8. In addition, we investigated how the relationship between home stay and MDD symptom severity was affected by participant age, gender, occupational status as well as by completeness and sampling constancy of the collected geolocation data. Moreover, we tested whether the strength of the relationship differed between weekdays and weekends.

The participants in the RADAR-MDD study were recruited from a nonhomogeneous population (ie, clinical and community samples with a wide age range) across 3 clinical sites in different European countries. When we pooled the data from all sites and used the entire biweekly segment before PHQ-8 completion, we found that home stay was positively associated with the PHQ-8 total score and age (Table 2). Specifically, the participants tended to spend more time at home with a greater severity of MDD symptoms and age. Furthermore, we found that occupational status was significantly related to home stay, with unemployed participants spending more time at home than their employed peers. Similar findings were observed when analyzing geolocation data collected over weekdays or weekends only, except for the association between home stay and the PHQ-8 total score (Table 2). The latter failed to reach statistical significance when tested with geolocation data of weekends only. This can be attributed to the ceiling effect [27], as the estimates of home stay obtained with geolocation data of the weekends were high for almost all participants at each individual site (Figure 3C; Multimedia Appendix 1, Figures S4 and S5 and Table S7). Although similar findings were observed for the KCL and CIBER sites, the association between home stay and the PHQ-8 total score did not reach statistical significance at the latter site (Multimedia Appendix 1, Table S8). This discrepancy could have been driven by participant recruitment primarily in a clinical setting and right skew of the PHQ- 8 total scores indicating great severity of the MDD symptoms in the participants recruited at the CIBER site (Multimedia Appendix 1, Figure S2). As the VUMC site recruited only 17 participants (Table 1) that, on average, exhibited mild to moderate symptoms of MDD (Multimedia Appendix 1, Figure S2), all the findings obtained with the data of that site only should be interpreted with caution.

\section{Comparison With Previous Work}

A variety of features can be extracted from geolocation data generated by smartphones and wearable devices and used to characterize the mobility patterns of an individual. These include home stay [15], the number of visited places [14], location entropy (ie, a metric that quantifies uniformity of the distribution of times spent by an individual at different locations) $[13,14]$, the maximal distance from home, and the total distance traveled [22]. Remarkably, several studies that investigated the relationship between mental health disorders and mobility patterns focused on home stay features $[12,22,28]$. In this study, we also used home stay to quantify the mobility patterns of the 
study participants, as home stay is considered an important indicator of social disengagement by clinicians [12]. Moreover, it has been demonstrated that home stay has a strong negative association with location entropy [9]. No features that quantify the distance traveled between visited locations (eg, the total distance traveled or the maximal distance from home) were used in our analysis, as the notion of distance was confounded by the fact that the participants lived in both urban and rural places and in different countries.

Several previous studies have documented a positive relationship between home stay and the severity of MDD symptoms [9,13-15]. To the best of our knowledge, no study, however, has collected data from either multiple sites or a nonhomogeneous population with a confirmed clinical diagnosis of MDD. Neither did those studies thoroughly address the factors of participants' age, occupational status, and data quality on the reported results. Furthermore, several previous studies analyzed data that were homogeneous in terms of the participant's age (ie, student population) $[12,24]$. In contrast, the age of the RADAR-MDD participants ranged from 18 to 73 years (Figure 2C). Our findings demonstrate that the strength of the relationship between home stay and the severity of MDD symptoms can be modulated by age. This relationship is expected to be stronger for younger individuals and weaker for older individuals, as the latter tend to stay at home more. In addition, all RADAR-MDD participants had a clinical diagnosis of MDD, and many of them had severe symptoms of MDD, as indicated by the high PHQ-8 total scores (Figure 2B). In contrast, participants from previous studies did not undergo clinical interviews and had overall low depression scores [11-14].

It has been pointed out $[9,22]$ that there exists no standard approach to the preprocessing of geolocation data generated by smartphones. Nonetheless, such important preprocessing steps, such as selection of an acceptable accuracy level and rate of missing data for the geolocation signal could have a significant impact on the reported results. In this study, we did not use all available geolocation data collected from the RADAR-MDD participants but instead applied stringent selection criteria (see Data Collection) to ensure high quality of the analyzed data and minimize the odds of reporting spurious results. In addition, we provide full and detailed information on the characteristics of the collected and analyzed geolocation data (Multimedia Appendix 1, Tables S1-S6).

\section{Limitations}

Although this was a multicenter study and the estimates of home stay were similar across the 3 sites, most participants were recruited at KCL (Multimedia Appendix 1, Figures S4 and S5 and Table S7). Several participants in the RADAR-MDD study followed antidepressant treatment, and some of them reported comorbidity with physical illness (eg, fibromyalgia). In addition, several participants were off sick or reported ill health. Antidepressants may cause a wide range of side effects, including headaches, fatigue, weight gain, drowsiness, and dizziness [29,30]. Individuals who experience any or all of these side effects or have comorbidities with physical illness are likely to spend more time at home than outdoors. This could have inflated the reported estimates of home stay (Figure 3) and thus distorted the strength of the relationship between home stay and overall severity of MDD symptoms.

Apart from medical and mental conditions, social factors may have also influenced how much time participants spent at home. These include the number of people living under the same roof and engaging in outdoor or community activities. The participants who were expected to assist their elderly family members in daily routines or take care of their children likely spent more time at home than their peers without such responsibilities. In contrast, engagement in outdoor or community activities, such as playing bingo or going to church, likely resulted in reduced home stay. Furthermore, it is commonly assumed that employment implies the physical presence of an employee in a designated workplace outside of home. However, we cannot rule out that some employed participants worked from home. Home teleworking likely increased home stay for those participants. As employment was significantly associated with reduced home stay in our data set, most employed participants in the study still worked outside their home. The effect of medication and physical comorbidity, social factors, and home teleworking on the relationship between daily mobility patterns and severity of MDD symptoms was beyond the scope of this study, although further research is warranted.

The stringent selection criteria imposed on completeness and sampling constancy of the collected geolocation data considerably reduced the number of biweekly segments available for analysis. Several factors could have affected the quality of the collected geolocation data. Poor mobile network coverage or weak GPS signals, for example, was expected to result in a higher missing rate of geolocation recordings. This was likely the case for participants living or traveling in distant or rural areas. Smartphone battery capacity could have constrained the total duration of the geolocation recordings. Owing to a limited battery capacity, frequent user interaction with a smartphone could have accelerated the battery drain and further limited the total duration of geolocation recordings. In addition, a high number of apps running in the background could have also contributed to a more rapid battery drain. The RADAR-MDD study was designed to concurrently collect a variety of data streams (eg, from a GPS sensor, a gyroscope, an accelerometer, a microphone, and an ambient light sensor embedded in a smartphone) to characterize the individual's behavior at full capacity [20,21]. This resulted in greater energy consumption and thus faster battery drain than in regular smartphones with no installed RADAR-MDD apps. Disabling the collection of one or multiple data streams in the study could have considerably prolonged the time smartphones operated on a single battery charge. Identification and comprehensive characterization of a single data stream or multiple data streams that convey most information on the individual's mental well-being is still a topic of active scientific research. Alternatively, event-driven collection of all or some data streams (eg, initiated by the individual's accidental movements or continuous motion) could have been less energy demanding than continuous sampling of those data streams as was implemented in the RADAR-MDD study. Finally, it is uncertain 
and requires additional examination whether the same results could have been obtained with more liberal selection criteria (eg, at least 10 instead of 14 days of recordings in a biweekly segment). If so, this would have increased the number of biweekly segments available for analysis and provided stronger evidence in support of the feasibility of geolocation data collection with smartphones.

\section{Conclusions}

We demonstrated that longer home stay can reflect greater symptom severity in individuals diagnosed with MDD. Although the relationship between home stay and MDD severity is modest, it can nonetheless improve remote monitoring of the individual's mental well-being, especially when combined with other informative correlates of MDD severity. However, it remains unclear whether the findings represent behavioral manifestations of MDD or are associated with changes in depressive symptoms. Additional analyses are required to test whether changes in home stay over time can be predictive of relapses in MDD. We also demonstrated that the relationship between home stay and MDD severity can be modulated by age, occupational status, and changes in daily routine. This finding is of great importance for a proper interpretation of similar studies conducted in the past and for better planning of future studies. Furthermore, our findings illustrate that passive remote monitoring of mobility patterns in individuals with MDD is feasible. This demonstrates the utility of smartphones and wearable devices with a GPS sensor in the collection of clinically relevant information that can be used to monitor the course of the disorder in a remote, unobtrusive, and ubiquitous manner, thus reducing patient burden and improving treatment.

\section{Acknowledgments}

The Remote Assessment of Disease and Relapse-Central Nervous System project has received funding from the Innovative Medicines Initiative 2 Joint Undertaking under grant agreement number 115902. This Joint Undertaking receives support from the European Union's Horizon 2020 research and innovation programme and European Federation of Pharmaceutical Industries and Associations [31]. This communication reflects the views of the Remote Assessment of Disease and Relapse-Central Nervous System consortium and neither Innovative Medicines Initiative nor the European Union and European Federation of Pharmaceutical Industries and Associations are liable for any use that may be made of the information contained herein. The funding body have not been involved in the design of the study, the collection or analysis of data, or the interpretation of data.

Participant recruitment in Amsterdam was partially accomplished through Hersenonderzoek.nl, a Dutch online registry that facilitates participant recruitment for neuroscience studies [32]. Hersenonderzoek.nl is funded by ZonMw-Memorabel (project number 73305095003), a project in the context of the Dutch Deltaplan Dementie, Gieskes-Strijbis Foundation, the Alzheimer's Society in the Netherlands and Brain Foundation Netherlands.

Participants in Spain were recruited through the following institutions: Parc Sanitari Sant Joan de Déu network of mental health services (Barcelona); Institut Català de la Salut primary care services (Barcelona); Institut Pere Mata-Mental Health Care (Tarrassa); Hospital Clínico San Carlos (Madrid).

This paper represents independent research part funded by the National Institute for Health Research (NIHR) Maudsley Biomedical Research Centre at South London and Maudsley National Health Service (NHS) Foundation Trust and King's College London. The views expressed are those of the authors and not necessarily those of the NHS, the NIHR, or the Department of Health and Social Care.

We thank all the members of the Remote Assessment of Disease and Relapse-Central Nervous System patient advisory board for their contribution to the device selection procedures, and their invaluable advice throughout the study protocol design.

This research was reviewed by a team with experience of mental health problems and their carers who have been specially trained to advise on research proposals and documentation through the Feasibility and Acceptability Support Team for Researchers: a free, confidential service in England provided by the National Institute for Health Research Maudsley Biomedical Research Centre via King's College London and South London and Maudsley NHS Foundation Trust.

We thank all Genetic Links to Anxiety and Depression Study volunteers for their participation, and gratefully acknowledge the NIHR BioResource, NIHR BioResource centres, NHS Trusts and staff for their contribution. We also acknowledge NIHR BRC, King's College London, South London and Maudsley NHS Trust and King's Health Partners. We thank the National Institute for Health Research, NHS Blood and Transplant, and Health Data Research UK as part of the Digital Innovation Hub Programme.

\section{Authors' Contributions}

PL and DAK extracted and integrated the questionnaire and geolocation data for the analysis, planned and performed the analysis, and drafted the manuscript. MH and VAN gained funding and co-led the Remote Assessment of Disease and Relapse-Central Nervous System program. MH is the principal investigator for the Remote Assessment of Disease and Relapse-Major Depressive Disorder study. RJBD, AAF, YR, ZR, PC, and CS have contributed to the development of the Remote Assessment of Disease and Relapse-based platform used for data collection and management across sites, data protection, security, and storage. PL, DAK, AAF, S Sun, YZ, FM, FL, S Siddi, BWJHP, JMH, PA, NC, SV, NVM, VAN, RJBD, and MH contributed to the design of the study. FM, AI, GL, and S Siddi collected the data. All authors meet the International Committee of Medical Journal Editors 
criteria, and all those who fulfilled these criteria are listed as authors. All authors have been involved in reviewing the manuscript, had access to the study data, provided direction and comments on the manuscript, made the final decision about where to publish these data, and approved submission to this journal. All authors agree with the content and author list of this manuscript.

\section{Conflicts of Interest}

DAK and NVM are employees of the Janssen Pharmaceutica NV and may hold company equity. SV and VAN are employees of Janssen Research and Development, LLC, and may hold company equity. PA is an employee of H. Lundbeck A/S and may hold company equity. MH declares research grants and in-kind contributions from Janssen, Biogen, UCB, MSD, and H. Lundbeck A/S through the Remote Assessment of Disease and Relapse-Central Nervous System consortium.

\section{Multimedia Appendix 1}

Tables and figures with information for each individual population site. [DOCX File, 509 KB-Multimedia Appendix 1]

\section{References}

1. Depression and other common mental disorders: global health estimates. World Health Organization. 2017. URL: https:/ lapps.who.int/iris/handle/10665/254610 [accessed 2021-12-30]

2. Cimpean D, Drake RE. Treating co-morbid chronic medical conditions and anxiety/depression. Epidemiol Psychiatr Sci 2011 Jun 08;20(2):141-150. [doi: 10.1017/s2045796011000345] [Medline: 21714361]

3. Lerner D, Adler DA, Chang H, Berndt ER, Irish JT, Lapitsky L, et al. The clinical and occupational correlates of work productivity loss among employed patients with depression. J Occup Environ Med 2004 Jun;46(6 Suppl):46-55 [FREE Full text] [doi: 10.1097/01.jom.0000126684.82825.0a] [Medline: 15194895]

4. Lenox-Smith A, Macdonald MT, Reed C, Tylee A, Peveler R, Quail D, et al. Quality of llfe in depressed patients in UK primary care: the FINDER study. Neurol Ther 2013 Dec 13;2(1-2):25-42 [FREE Full text] [doi: 10.1007/s40120-013-0006-1] [Medline: 26000214]

5. Cuijpers P, Schoevers RA. Increased mortality in depressive disorders: a review. Curr Psychiatry Rep 2004 Dec;6(6):430-437. [doi: 10.1007/s11920-004-0007-y] [Medline: 15538991]

6. Callan JA, Wright J, Siegle GJ, Howland RH, Kepler BB. Use of computer and mobile technologies in the treatment of depression. Arch Psychiatr Nurs 2017 Dec;31(3):311-318. [doi: 10.1016/j.apnu.2016.10.002] [Medline: 28499574]

7. Grist R, Porter J, Stallard P. Mental health mobile apps for preadolescents and adolescents: a systematic review. J Med Internet Res 2017 May 25;19(5):e176 [FREE Full text] [doi: 10.2196/jmir.7332] [Medline: 28546138]

8. Mohr DC, Zhang M, Schueller SM. Personal sensing: understanding mental health using ubiquitous sensors and machine learning. Annu Rev Clin Psychol 2017 May 08;13:23-47. [doi: 10.1146/annurev-clinpsy-032816-044949] [Medline: $\underline{28375728]}$

9. Rohani DA, Faurholt-Jepsen M, Kessing LV, Bardram JE. Correlations between objective behavioral features collected from mobile and wearable devices and depressive mood symptoms in patients with affective disorders: systematic review. JMIR Mhealth Uhealth 2018 Aug 13;6(8):e165 [FREE Full text] [doi: 10.2196/mhealth.9691] [Medline: $\underline{30104184]}$

10. Liang Y, Zheng X, Zeng DD. A survey on big data-driven digital phenotyping of mental health. Inform Fusion 2019 Dec;52:290-307. [doi: 10.1016/j.inffus.2019.04.001]

11. Canzian L, Musolesi M. Trajectories of depression: unobtrusive monitoring of depressive states by means of smartphone mobility traces analysis. In: Proceedings of the 2015 ACM International Joint Conference on Pervasive and Ubiquitous Computing. 2015 Presented at: UbiComp '15: The 2015 ACM International Joint Conference on Pervasive and Ubiquitous Computing; September 7 - 11, 2015; Osaka Japan p. 1293-1304. [doi: 10.1145/2750858.2805845]

12. Chow PI, Fua K, Huang Y, Bonelli W, Xiong H, Barnes LE, et al. Using mobile sensing to test clinical models of depression, social anxiety, state affect, and social isolation among college students. J Med Internet Res 2017 Mar 03;19(3):e62 [FREE Full text] [doi: 10.2196/jmir.6820] [Medline: 28258049]

13. Saeb S, Zhang M, Karr CJ, Schueller SM, Corden ME, Kording KP, et al. Mobile phone sensor correlates of depressive symptom severity in daily-life behavior: an exploratory study. J Med Internet Res 2015 Jul 15;17(7):e175 [FREE Full text] [doi: 10.2196/jmir.4273] [Medline: 26180009]

14. Saeb S, Lattie EG, Schueller SM, Kording KP, Mohr DC. The relationship between mobile phone location sensor data and depressive symptom severity. PeerJ 2016;4:e2537 [FREE Full text] [doi: 10.7717/peerj.2537] [Medline: 28344895]

15. Wahle F, Kowatsch T, Fleisch E, Rufer M, Weidt S. Mobile sensing and support for people with depression: a pilot trial in the wild. JMIR Mhealth Uhealth 2016 Sep 21;4(3):e111 [FRE Full text] [doi: 10.2196/mhealth.5960] [Medline: 27655245]

16. Matcham F, di San Pietro CB, Bulgari V, de Girolamo G, Dobson R, Eriksson H, RADAR-CNS Consortium. Remote assessment of disease and relapse in major depressive disorder (RADAR-MDD): a multi-centre prospective cohort study protocol. BMC Psychiatry 2019 Feb 18;19(1):72 [REE Full text] [doi: 10.1186/s12888-019-2049-z] [Medline: 30777041 ] 
17. RADAR-CNS: Remote Assessment of Disease and Relapse - Central Nervous System. RADAR-CNS. URL: http://radar-cns. org/ [accessed 2021-05-23]

18. Kroenke K, Strine TW, Spitzer RL, Williams JB, Berry JT, Mokdad AH. The PHQ-8 as a measure of current depression in the general population. J Affect Disord 2009 Apr;114(1-3):163-173. [doi: 10.1016/j.jad.2008.06.026] [Medline: 18752852]

19. Bot M, Middeldorp CM, de Geus EJ, Lau HM, Sinke M, van Nieuwenhuizen B, et al. Validity of LIDAS (LIfetime Depression Assessment Self-report): a self-report online assessment of lifetime major depressive disorder. Psychol Med 2016 Oct 05;47(2):279-289. [doi: 10.1017/s0033291716002312]

20. Ranjan Y, Rashid Z, Stewart C, Conde P, Begale M, Verbeeck D, Hyve, RADAR-CNS Consortium. RADAR-Base: open source mobile health platform for collecting, monitoring, and analyzing data using sensors, wearables, and mobile devices. JMIR Mhealth Uhealth 2019 Aug 01;7(8):e11734 [FREE Full text] [doi: 10.2196/11734] [Medline: 31373275]

21. RADAR-base: Remote Assessment of Disease and Relapse - an mHealth Platform for Generalized Data Collection. RADAR-base. URL: http://radar-base.org/ [accessed 2021-05-23]

22. Depp CA, Bashem J, Moore RC, Holden JL, Mikhael T, Swendsen J, et al. GPS mobility as a digital biomarker of negative symptoms in schizophrenia: a case control study. NPJ Digit Med 2019 Nov 08;2(1):108 [FREE Full text] [doi: 10.1038/s41746-019-0182-1] [Medline: $\underline{\text { 31728415] }}$

23. Barnett I, Torous J, Staples P, Sandoval L, Keshavan M, Onnela J. Relapse prediction in schizophrenia through digital phenotyping: a pilot study. Neuropsychopharmacology 2018 Jul;43(8):1660-1666. [doi: 10.1038/s41386-018-0030-z] [Medline: 29511333]

24. Farhan A, Lu J, Bi J, Russell A, Wang B, Bamis A. Multi-view bi-clustering to identify smartphone sensing features indicative of depression. In: In Proceedings of the IEEE First International Conference on Connected Health: Applications, Systems and Engineering Technologies (CHASE). 2016 Presented at: IEEE First International Conference on Connected Health: Applications, Systems and Engineering Technologies (CHASE); June 27-29, 2016; Washington, DC, USA p. 264-273. [doi: 10.1109/chase.2016.27]

25. Farhan A, Yue C, Morillo R, Ware S, Lu J, Bi J, et al. Behavior vs. introspection: refining prediction of clinical depression via smartphone sensing data. In: Proceedings of the IEEE Wireless Health (WH) Conference. 2016 Presented at: IEEE Wireless Health (WH) Conference; Oct. 25-27, 2016; Bethesda, MD, USA p. 1-8. [doi: 10.1109/wh.2016.7764553]

26. Albert P. Why is depression more prevalent in women? J Psychiatry Neurosci 2015 Jul 1;40(4):219-221 [FREE Full text] [doi: 10.1503/jpn.150205] [Medline: 26107348]

27. Šimkovic M, Träuble B. Robustness of statistical methods when measure is affected by ceiling and/or floor effect. PLoS One 2019 Aug 19;14(8):e0220889 [FREE Full text] [doi: 10.1371/journal.pone.0220889] [Medline: $\underline{31425561]}$

28. Tung JY, Rose RV, Gammada E, Lam I, Roy EA, Black SE, et al. Measuring life space in older adults with mild-to-moderate Alzheimer's disease using mobile phone GPS. Gerontology 2014;60(2):154-162. [doi: 10.1159/000355669] [Medline: 24356464]

29. Ferguson JM. SSRI antidepressant medications: adverse effects and tolerability. Prim Care Compan J Clin Psychiatry 2001 Mar 01;3(1):22-27 [FREE Full text] [doi: 10.4088/pcc.v03n0105] [Medline: 15014625]

30. Wang S, Han C, Bahk W, Lee S, Patkar AA, Masand PS, et al. Addressing the side effects of contemporary antidepressant drugs: a comprehensive review. Chonnam Med J 2018 May;54(2):101-112 [FREE Full text] [doi: 10.4068/cmj.2018.54.2.101] [Medline: 29854675]

31. Europe's partnership for health. Innovative Health Initiative. URL: https://www.imi.europa.eu/ [accessed 2022-01-11]

32. Hersenonderzoek. URL: https://hersenonderzoek.nl/ [accessed 2022-01-11]

\section{Abbreviations}

CIBER: Centro de Investigación Biomédica en Red

KCL: King's College London

MDD: major depressive disorder

PHQ-8: 8-item Patient Health Questionnaire

RADAR-MDD: Remote Assessment of Disease and Relapse-Major Depressive Disorder

RMT: remote monitoring technology

VUMC: Vrije Universiteit Medisch Centrum 
Edited by L Buis; submitted 21.02.21; peer-reviewed by A Baglione, A AL-Asadi, D Mack, W Chan; comments to author 27.04.21; revised version received 20.06.21; accepted 21.10.21; published 28.01 .22

Please cite as:

Laiou P, Kaliukhovich DA, Folarin AA, Ranjan Y, Rashid Z, Conde P, Stewart C, Sun S, Zhang Y, Matcham F, Ivan A, Lavelle G, Siddi S, Lamers F, Penninx BWJH, Haro JM, Annas P, Cummins N, Vairavan S, Manyakov NV, Narayan VA, Dobson RJB, Hotopf $M, R A D A R-C N S$

The Association Between Home Stay and Symptom Severity in Major Depressive Disorder: Preliminary Findings From a Multicenter Observational Study Using Geolocation Data From Smartphones

JMIR Mhealth Uhealth 2022;10(1):e28095

URL: https://mhealth.jmir.org/2022/1/e28095

doi: $10.2196 / 28095$

PMID:

CPetroula Laiou, Dzmitry A Kaliukhovich, Amos A Folarin, Yatharth Ranjan, Zulqarnain Rashid, Pauline Conde, Callum Stewart, Shaoxiong Sun, Yuezhou Zhang, Faith Matcham, Alina Ivan, Grace Lavelle, Sara Siddi, Femke Lamers, Brenda WJH Penninx, Josep Maria Haro, Peter Annas, Nicholas Cummins, Srinivasan Vairavan, Nikolay V Manyakov, Vaibhav A Narayan, Richard JB Dobson, Matthew Hotopf, RADAR-CNS. Originally published in JMIR mHealth and uHealth (https://mhealth.jmir.org), 28.01.2022. This is an open-access article distributed under the terms of the Creative Commons Attribution License (https://creativecommons.org/licenses/by/4.0/), which permits unrestricted use, distribution, and reproduction in any medium, provided the original work, first published in JMIR mHealth and uHealth, is properly cited. The complete bibliographic information, a link to the original publication on https://mhealth.jmir.org/, as well as this copyright and license information must be included. 